\title{
Transit networks, social contacts and open data meet public transportation plans for post-COVID-19: A Canadian case study
}

\author{
Oliver Benning, Jonathan Calles, Burak Kantarci and Shahzad Khan
}

\begin{abstract}
The emergence of COVID-19 and its variants has dramatically shifted the way that societies respond to a pandemic crisis. One of the response needs exposed is the ability for cities to track potential transmission of contagions within population movements effectively, which can exploit the means of pervasive computing by collecting and processing ubiquitously acquired data, as well as long term data that constitutes prior but limited contextual knowledge. Thus, bridging the cyber and physical aspects in the resolution of COVID-19 crisis, as well as post-COVID19 is an emerging field of research and development. This article presents a practical method for the assessment of the risk profiles of communities by tracking / acquiring, fusing and analyzing data from public transportation, district population distribution, passenger interactions and cross-locality travel data. The proposed framework fuses these data sources into a realistic simulation of a transit network for a given time span. By shedding credible insights into the impact of public transit on pandemic spread, the research findings will help to set the groundwork for tools that could provide pandemic response teams and municipalities with a robust framework for the evaluations of city districts most at risk, and how to adjust municipal services accordingly.
\end{abstract}

Index Terms-COVID-19, post-COVID-19 management, pandemic, public transportation, general transit feed specification, social contacts, decision support

\section{INTRODUCTION}

COVID-19 has both symptomatic and asymptomatic responses, rendering it difficult to track in communities [1]. A year after the initial COVID-19 outbreak, it has become clear the impact that widespread epidemics can have impacts on both the supply and demand of economic activity and government services [2], [3]. There has been a greater sense of importance given to tracing population behaviour and preferences in cities, to better understand the pandemic, as well as pressing questions on how to strike a balance between safeguarding public health while resuming regular life activities [4]-[6].

Early in the pandemic the Center for Disease Control and Prevention (CDC) issued general guidance on adjusting public bus routes between areas experiencing different levels of transmission [7]. It is worth to note that thorough research and investigation is required to support these decisions. Equally,

O. Benning, J. Calles and B. Kantarci are with the School of Electrical Engineering and Computer Science at the University of Ottawa, Ottawa, ON, K1N 6N5, Canada.

Emails: \{obenn009,jcall057,burak.kantarci@uottawa.ca\}

S. Khan is with Gnowit Inc., 308 Legget Dr, Kanata, ON K2K 1Y6, Canada Email: shahzad@gnowit.com societies have started to tackle the question of whether public transportation triggers high transmission rates, and more importantly whether public transportation can still be relied on by the public in a post-COVID-19 world [8]. It is worth to note that established organizations such as the American Public Transportation Association report no direct correlation between COVID-19 transmission and urban public transit [9]. On the other hand, research on the development of policies and procedures on how to use public transport during and after the pandemic has reported solid guidelines [10], [11].

In this article, we aim to assist the decision makers at the municipal level regarding public transport planning in a post-COVID-19 world by respecting the privacy of commuters and communities. To do so, we investigate the role of public transportation as a transmission vector during pandemics. Public research often lacks the necessary data to properly evaluate transit systems in this context, due to privacy rights and limitations on the collection of mobility data for commuters. We document our case study in Ottawa (Ontario, Canada) through analysis which instead fuses readily available (and de-anonymized) data, i.e., General Transit Feed Specification (GTFS), Census, and transit ridership levels tracked by Apple.

By fusing these data, we introduce a ready-to-use simulation framework to create a representation of a public transportation network capable of modelling transit routes, commuter behaviour, and passenger interactions. With a few inputs requirements (which could be based on reasonable assumptions), we simulate and model with granular detail on the number, time and whereabouts (i.e., specific ward in the city) of person-toperson contacts.

The analysis and findings in this article detail how the results of this approach identify inter-ward activity by capturing data on ward visitations and cumulative commuter interactions. By analysing the results for Ottawa, we are able to explain how this methodology could enable city administrators and public health officials to simulate the spread of any outbreak (including COVID-19 and potential variants) within a city, eventually leading to broader decisions such as service adjustments amid pandemics.

The contribution of this article is to present a data-driven simulation platform to contribute to the addressing the engineering management challenges towards a post-COVID-19 world through:

- Modelling person-to-person interactions on public trans- 
portation systems,

- Evaluation of community risk profiles based on connectivity to public transportation networks and commuter participation,

- A framework for safely evaluating public transportation service offerings in pandemic scenarios,

- Insights into the correlation between public transit ridership and COVID-19 infection rates by presenting a case study in Canada.

The next sections are formulated as follows: Section II presents the related work on public transportation and contagion transmission modelling research. Section III presents the design principles for the proposed simulation platform. Section IV details the simulator architecture. Section V presents a case study and analyses on the simulator outputs. Section VI presents the open issues, discussions, and lessons learned whereas Section VII concludes the article.

\section{RElated WORK AND StATE OF THE ART}

As of July 2020, researchers in [12] have identified a need for further investigation on modelling the transmission of COVID19 through public transportation, and leveraging contact tracing to prevent the disease from spreading in an uncontrolled manner. Studies of public transportation networks focus on system infrastructure with higher emphasis on optimization of route efficiency by leaving room for future research on passenger activity [13] and capacity planning of the public transportation vehicles [14], as well as the use of autonomous robots to deliver several services [15]. In addition to these, research on the readiness / preparedness of public transportation systems against pandemics has become critical [16].

While simulators are capable of emulating passenger behaviour require private or proprietary data sets in addition to GTFS data, such as fare validation statistics [17], there exists research that relies on only GTFS data and self-sourced crowdsensing data in the context of public transportation networks [18]. Indeed, integrating this type of research with passenger interactions would be invaluable for public health. Nevertheless, mobile crowd-sensing provides a promising venue to study community risk factors during pandemics [19].

It is foreseeable that crowd-sensing could be applied to public transportation systems (e.g., [20]). With this in mind, it might be possible to identify passenger interaction clusters. However, selfsourced data of this type would not be available immediately. With this in mind, we take a novel approach to simulate public transportation using already available data as a means to model commuter behaviour and in turn potential transmission vectors during the pandemic and in the post-COVID-19 era.

\section{DESIGN PRINCIPLES}

We present the underlying simulator framework to be as flexible as possible for users in regard to their choice of city, and achievable with respect to data availability.

\section{A. Leveraging open data}

We combine widely available data sets for transit feeds, census data, and municipality subdivision to structure the simulator. In Canada (and most cities in the U.S.) the consistent format of the data allows the simulator to be run on almost every major Canadian city with no or slight modifications. It is worth to note that if a census is not available, an alternate source of population ward data and ridership can be provided.

The General Transit Feed Specification (GTFS) standard defines a common standard for public transport geographic information as well as scheduling information such as routes and trips. We use this data to obtain information about the layout of the transit system, as well as precise schedules for the simulations. Furthermore, as city boundaries are normally derived from population metrics, they provide a good basis for defining subjective communities within a city during pandemic scenarios.

Amongst the national census data collected is population, employment status, habits and other demographic or socioeconomic statistics. Canadian data is centralized and available on the Statistics Canada website [21]. This makes census data a convenient input for our simulator and allows application to almost any Canadian city with minimal modifications. The Canadian census further collects the numbers on primary means of commuting, car, bicycle, public transit, as well as commute times and other temporal trends. These aggregated data enables us to realistically model attributes of passengers on public transportation such as peak rush hour commutes.

\section{B. Realistic Population Behaviour}

We utilise the population data from the census, as well as mobility data from Apple to realistically spawn commuters at different bus stops in the system. From there, we utilize the average commuter trip in kilometers, as obtained from the transit agency, as well as the GTFS schedule data, to simulate these riders as they propagate through the system.

\section{Community Risk Profiling}

Our goal is to determine regional risk profiles over time due to transmission spread between communities. The model therefore tracks interactions between the virtual passengers and scores each time interval over the course of a day with the sum of interactions from passengers who got off during that time interval in a particular ward. The use of predetermined ward demarcations based on electoral districts, provides an objective definition of a 'community' within this tracking context, and helps to provide a partition of the city, so it can be identified which parts contain the highest passenger-interaction index.

Infection rates are a function of transmission, as the tracked movement determines the risk profile of communities by simulating exposure to the virus. Thus, public transportation units can monitor whether their current operational decisions impact community infection rates based on current public transportation usage. 


\section{Simulator Architecture}

The Python-based simulator uses data from the city of Ottawa for analyses and validation; however, this architecture could apply to any city with appropriate data.

\section{A. GTFS data}

The public transit agency of Ottawa (i.e., OC Transpo) provides both GTFS static data, and real-time data feeds. The static data that describes the transport topography is publicly available and conforms to the GTFS standard. Figure 1 compares the OC Transpo data against the requirements of GTFS.

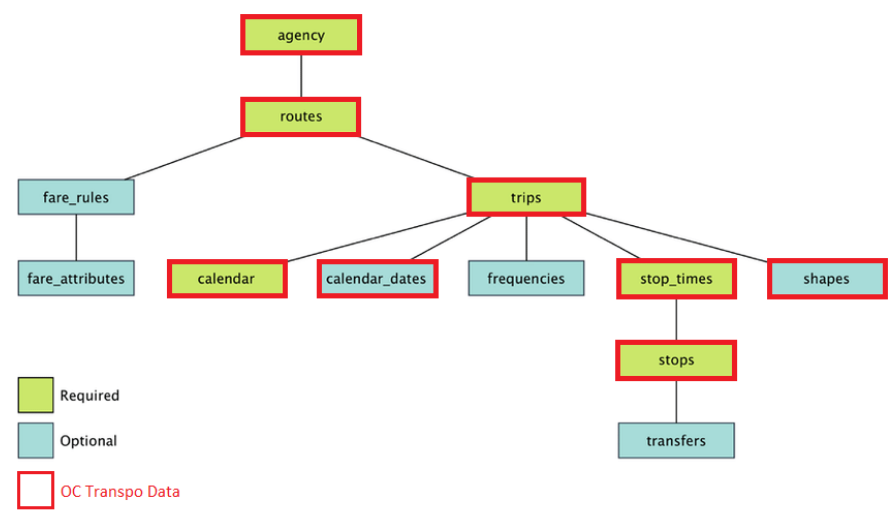

Fig. 1: Availability of OC Transpo data against GTFS standard

Although disjoint, the GTFS data provides plenty of identifying information, as well as sufficient information to reconstruct the spatial-temporal configuration of the public transit network. The GFTS data that needs to be used is limited to the data that can help to form a flow graph that represents the transit network, i.e., scheduled trips and stops for the bus routes.

\section{B. Municipal Ward Data}

As pointed out by the research on COVID-19, the availability and usability of open data is essential for public health organizations to effectively respond to the pandemic [22], [23]. With this in mind, the availability of ward data of cities is of paramount importance. In the case of Ottawa, the municipality is sub-divided into electoral districts known as wards. The City of Ottawa's 'Open Ottawa' project provides open-source data about the city including the geographical boundaries for these wards. In this research, we use the 2010 ward boundaries, which are publicly available [24].

\section{Census and Mobility Data}

Canadian Census for Ottawa [21] provides population data for our model. The "main mode of commuting" section in the census data is used to identify the use of public transportation on a daily basis during peak commute times. The section titled "time leaving for work" in the census data motivates the use

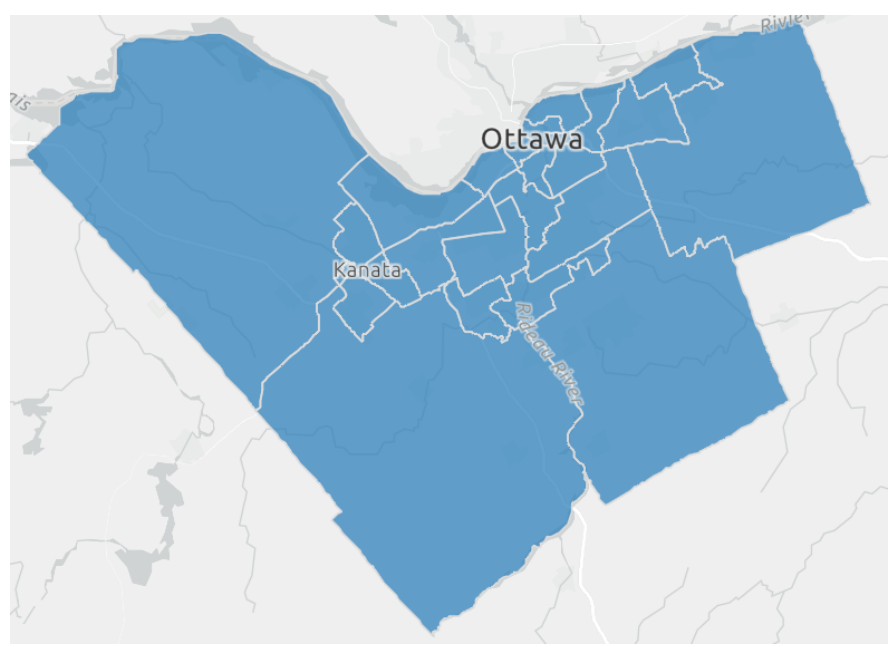

Fig. 2: Ottawa ward boundaries (Information is publicly available in open.ottawa.ca )

of a normal distribution to model rush hours and provides a reference mean and standard deviation to use. Notably 8am with a standard deviation of 2 hours, it can be assumed that the return time would be centered around $4 \mathrm{pm}$ due to the commonality of an 8-hour workday with certain exceptions.

\section{Generating a Graph-Based Model}

The underlying structure of the simulator is a graph-based representation of the GTFS data as simply illustrated in Fig. 3. Specifically, a directed weighted graph where vertices represent bus stops, and hold geo-location information for later correlation with ward data. Directed edges represent stops linked by a bus trip, and the weight of the edge is defined by the time taken for a bus to travel between the two scheduled stops. Generating the graph-based structure follows a simple methodology: Initially, for each bus stop in the GTFS data, a vertex object is added to the graph. Next, by iterating through all the bus trips within the selected time frame, for each pair of adjacently scheduled stops, an edge is added to the graph to connect the vertices representing the respective stops. The weight of the edges are assigned the time difference between the scheduled stop times.

As the graph-based uses bus stops for vertices, corresponding to physical locations, this allows for tracking transit patterns with respect to geographical vectors. Incidents within graph representation can serve as indicators feeding directly into community risk factors based on location. Figure 4 illustrates snapshots of the resulting graph object for two days of the week.

The contrast between the two, show how graph object can represent the transit system at different points in time.

\section{E. Design of simulator}

By using the graph-based representation of the GTFS data, the system is added the capacity to take in flexible algorithms 


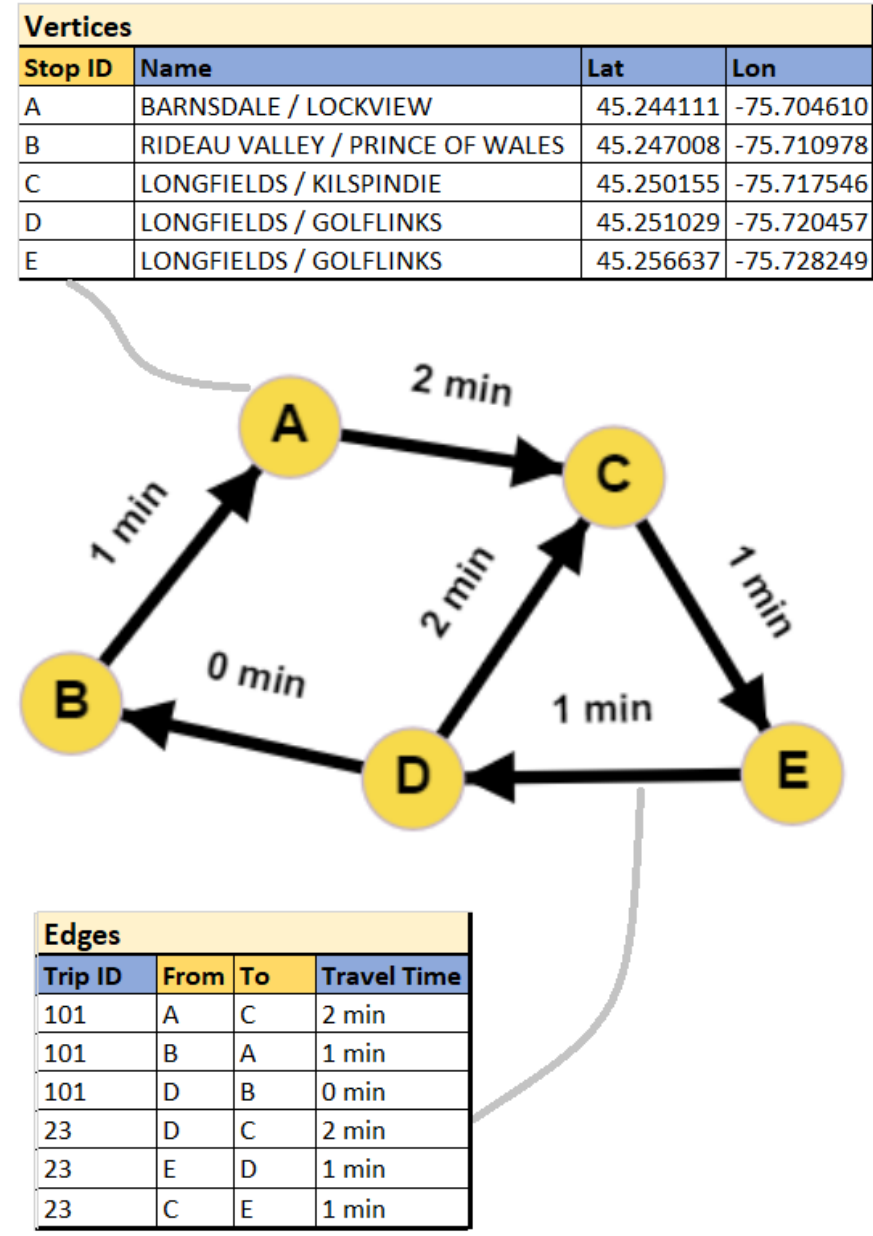

Fig. 3: Simple illustration graph generation

for the distribution of passengers who enter the system, and whether they perform actions such as transferring or getting off of a bus. This is followed by the implementation of these algorithms that creates an accurate simulation in an otherwise chaotic system. This brings the advantage of being able to model the interactions between different aspects of the system including riders, buses and wards during a given time-frame. The simulator offers the ability to track passenger interactions with total precision and with respect to location where most bus network data or simulators do not track the interactions of riders with the same degree of precision.

The time progression of the model is in intervals representing 1 minute to accurately emulate the progression of bus vehicles travelling their routes. The simulator therefore contains two main stages: 1. Pre-processing of the graph structure to populate data and parameters, 2. Executing a tick for each minute in the given time-frame and performing one step of the simulation.

The simulator takes parameters described in Table I, and the day being simulated is determined in the graph generation phase.

\section{Friday}

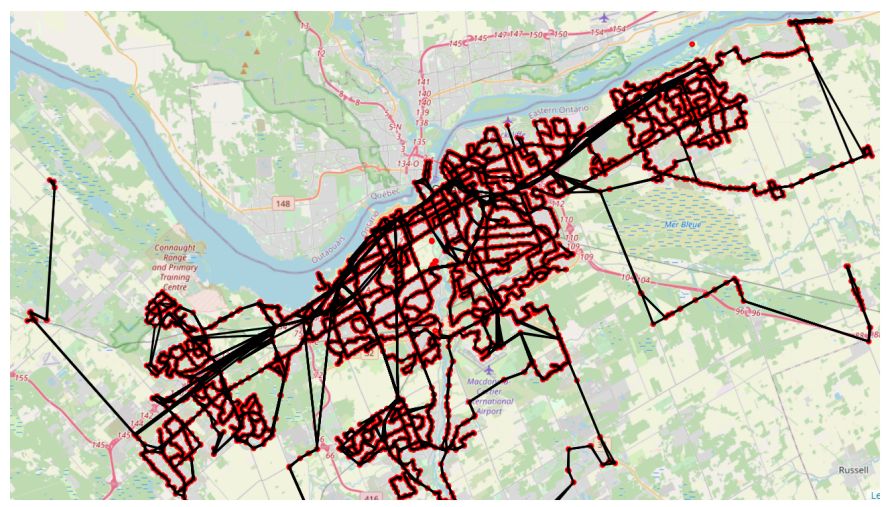

Sunday

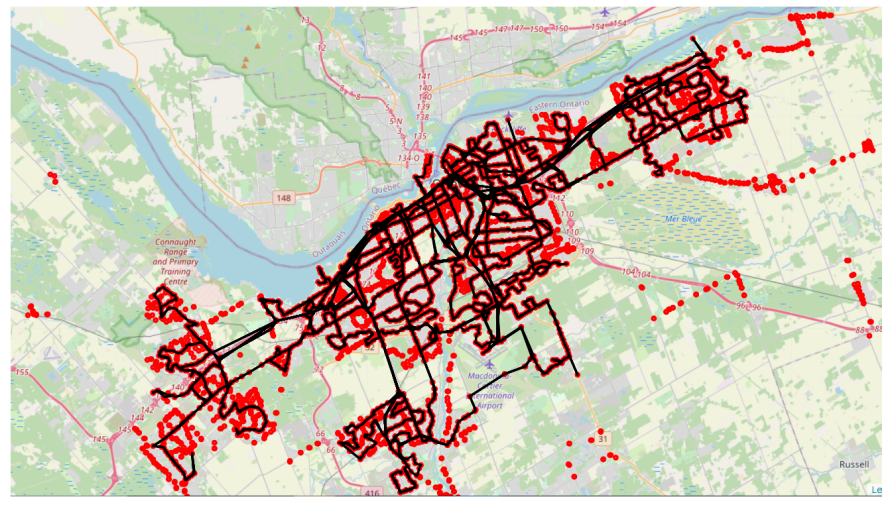

Fig. 4: Visualization of graph representation, Friday and Sunday

$\begin{array}{lll}\text { Parameter } & \begin{array}{l}\text { Description } \\ \text { time to start the simulator }\end{array} & \begin{array}{l}\text { Default } \\ \text { start }\end{array} \\ \text { time to stop the simulator } & 23: 00: 00 \\ \text { end } & \text { bus departure function } & \text { built-in } \\ \text { process_on } & \text { bus arrival function } & \text { built-in } \\ \text { process_off } & \text { minutes per result bucket } & 30 \\ \text { granularity } & \text { passenger travel distance } & 10 \\ \text { kms_mean } & \text { passenger travel variation } & 5 \\ \text { kms_stddev } & \text { passenger transferability } & \text { True } \\ \text { transfers } & \text { morning ridership peak } & 08: 00: 00 \\ \text { am_rush_hr } & \text { afternoon ridership peak } & 16: 00: 00 \\ \text { pm_rush_hr } & \text { stddev for am peak } & 2 \\ \text { am_rush_stddev } & \text { stddev for pm peak } & 2\end{array}$

TABLE I: Simulator parameters

To emulate regular commuter patterns with peak rush hours, passengers generated to enter the system using a bi-modal distribution, i.e., two normal distributions: one for the morning rush hour, and one for the afternoon rush hour using the provided means and standard deviation in the parameters. The concatenation of the two distributions forms a representation of daily ridership.

To match real life as closely as possible one of these distributions is generated for each ward, with peaks proportional to the ward's transit regular ridership levels adjusted by Apple's mobility index for transit ridership. This adjustment is an important way to account for declined ridership during the 
COVID-19 pandemic [25].

The final output of the simulator is a matrix of size $W \times B$ where $W$ is the number of wards and $B$ is the number of timeframe buckets, obtained by the equation (end - start)/granularity, where each bucket represents the number of interactions recorded in each window in granularity minutes. For example, with a time frame of 03:00:00 - 23:00:00 there are 20 hours, and if granularity is set to 30 , we would have 40 buckets, one for each interval of 30 minutes from the start to the end.

\section{CAse Study and Analyses}

Prior to presenting the analyses, it is worth to present the runtime overhead of the presented simulations. The full run-time is $O\left(W+C+D+T^{2}+S^{2}\right)$ where $S$ is the number of stops in the GTFS data, $W$ is the number of wards, $C$ denotes the number of GTFS calendar entries in the data and $D$ represents the number of GTFS calendar_dates in the data, and $T$ is the size of the GTFS stop_times data. For reference, processing the graph for a day in Ottawa takes around 8 minutes.

\section{A. Simulation Process}

In order to gain insight into the data for Ottawa, we run the simulations 10 times on on a granularity of 30 minutes for July 2nd, 2020, and plot the results for all runs over each ward, along with the mean. This is with the intent of visualizing the risk of each ward over the time of day, along with the variability. We utilize a mean of $10 \mathrm{~km}$ and a standard deviation of $6 \mathrm{~km}$ for calculating the approximate average passenger trip length for the first set of simulations.

From the associated graphs we can see the overall trends for each ward. In Fig. 5, the output for the Kanata South, a relatively suburban ward, the peak of the contacts occur between $3 \mathrm{pm}$ and $5 \mathrm{pm}$, which coincide with the time to return home from work. A corresponding spike does not exist for the morning commute, as during these hours people would be leaving the "transmitter" ward, and those interactions would be tracked as metrics of the "receptor" wards.

When analysing a central down-town ward like Rideau-Vanier in Fig. 6, we see much larger spread in contact density, and a second peak in the morning. This makes sense for a ward close to downtown / city center where inbound and outbound traffic reach their peak during rush hour commutes, as well as considerable activity throughout the day as well.

\section{B. Analyses with respect to COVID-19 infection rates}

In order to figure out the correlation between the cumulative contacts generated for each ward over the day and actual infections of COVID-19, we compare our results to the open data platform for the tracking of COVID-19 infections in Ottawa, provided by the city of Ottawa [26]. For each of the analyses we run a simulation using 10 repetitions, and 3 sets of values for the mean and standard deviation in kilometers of the passenger

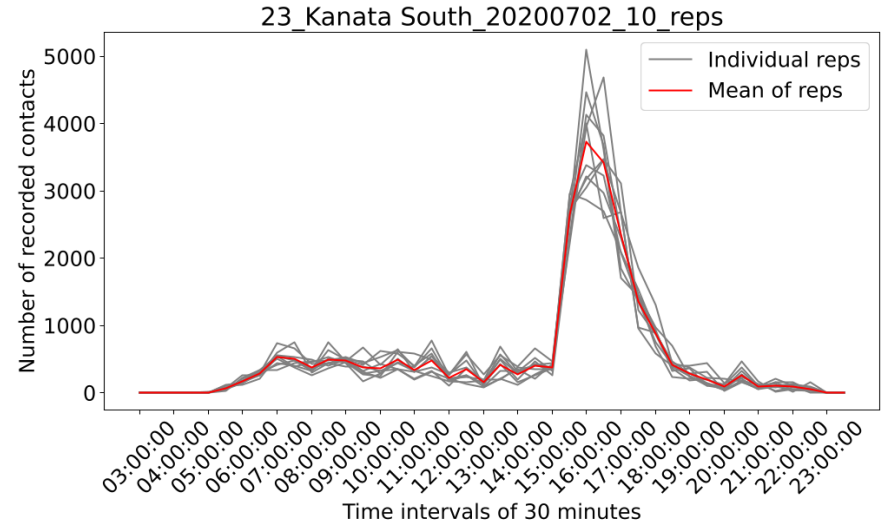

Fig. 5: Recorded contacts in Kanata South ward

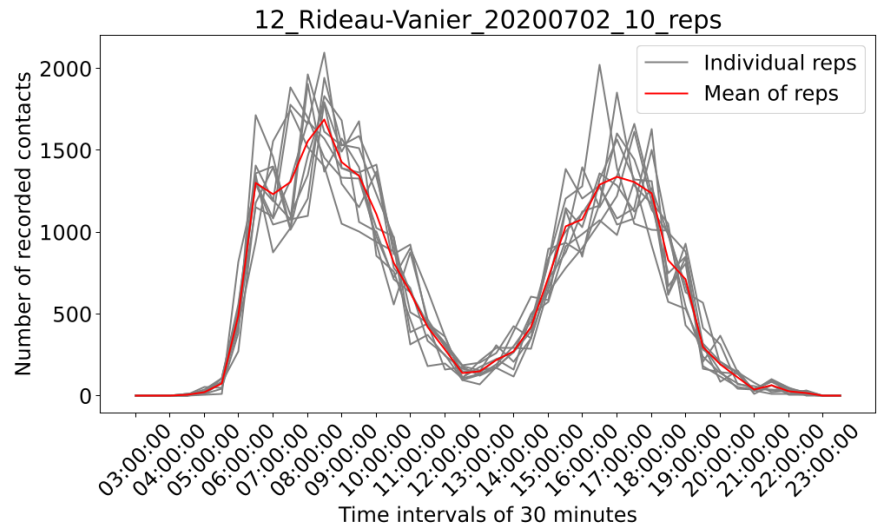

Fig. 6: Recorded contacts in Rideau Vanier ward

travel distance. The values are $5 \mathrm{~km}$ mean with $3 \mathrm{~km}$ standard deviation, $10 \mathrm{~km}$ mean with $6 \mathrm{~km}$ standard deviation and $10 \mathrm{~km}$ mean with $3 \mathrm{~km}$ standard deviation respectively. The rational behind the 3 scenarios is to see if the correlations observed vary at all with respect to ridership distance.

First, ward populations and ward infection rates are investigated as a baseline estimator. This comparison points out a statistically significant correlation index of 0.597 , as shown in Fig. 7.

When compared with the per ward cumulative infection rate on December 5th, 2020 (excluding long-term care home infections) using the Pearson $r$ test, a statistically insignificant correlation coefficient of $0.216,0.056$ and 0.04 is obtained respectively in each test as seen in Figs. 8a, 8b and 8c. Assuming the simulation to be realistic, these results show that the Ottawa public transit network does not contribute a statistically significant amount to the spread of COVID-19 between wards. This is in-line with the latest advice of the American Public Transportation Association report in [9], which states that a correlation between public transit and transmittal of the virus is unlikely. 


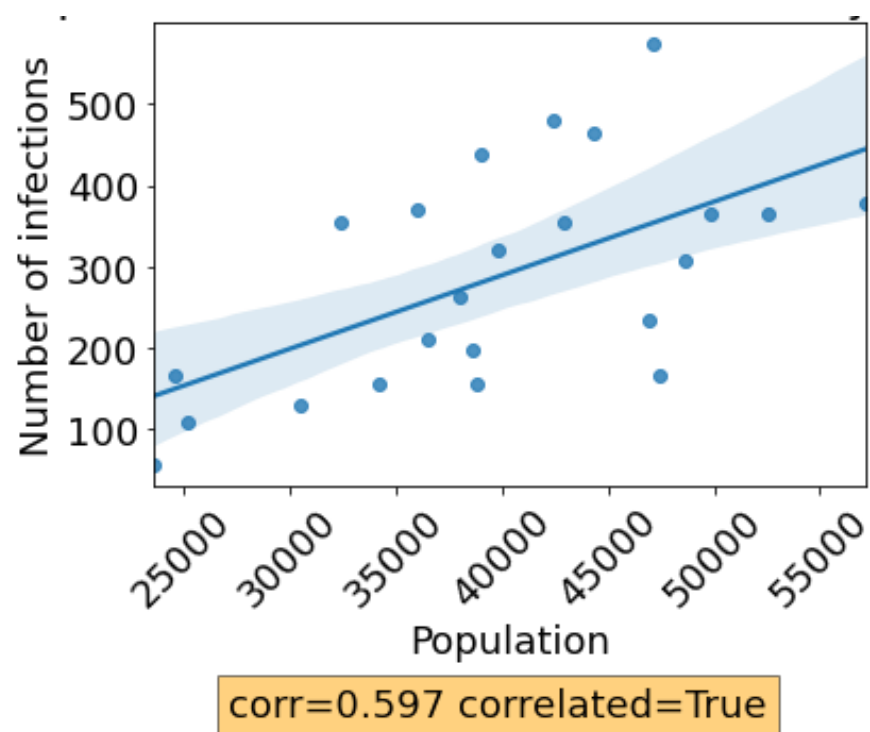

Fig. 7: Ward populations vs real infection rates by ward. Each point on the plot represents the number of infected individuals in a particular ward with its corresponding population on the $\mathrm{x}$-axis.

\section{Transit contact analysis}

With the conclusion that the transit contact index is not correlated to infection rates, we investigate the major factor(s) that may affect the transit contact index significantly. Since the data reveals that wards closer to downtown get more contacts, we compare values from the transit context index for each ward with its proximity to the city center in kilometers. As seen in Figs. 9a, 9b and 9c, a ward's distance from the city center correlates inversely with the number of simulated transit contacts in a statistically significant magnitude. Therefore, this study can conclude that the number of transit contacts a ward produces is related to how close it is to the city center, which makes intuitive sense as most commute is from residential areas to downtown and back. While Ottawa's public transit network does not seem to pose an infection risk, such comparison metrics could help a city adjust its network if a risk was ever found.

\section{OpPortunities AND OPEN ISSUES TO ADDRESS IN A POST-COVID-19 WORLD}

In this section we present various directions that this work could be taken to contribute to different areas of research. Some of the ideas listed here motivate the original development of the approach taken in this research.

\section{A. Advice for municipalities}

While public transit alone, and the identification of communities with a high contact indexes, do not prove to be a good predictor of infection rates, availability of this simulator enables identifying hot spots of commuter interactions. In a postCOVID-19 world, the simulator-based approach could be used to safely make assessments of service adjustments to public transportation systems, to either minimize the possibility of transmission or adjusting to a 'new normal' of pandemics [27] as experienced during COVID-19. The framework could also help to design new routes to reduce personal contacts. Graphs for certain wards show clear spikes in traffic, where the number of busses could be increased to help smooth out the contact rates amongst individuals. Additionally, contact is a measure of how crowded public vehicles are, and this could be used to address the general problem of avoiding overcrowding in public transit.

\section{B. Generalizing to other municipalities}

While Ottawa-based case study outlined in this article shows no correlation with infection rates of the city, Ottawa is sparse city with moderate public transit ridership. When the simulations are run and further improved by applying them in different cities, such as denser ones like Toronto where public transportation plays a bigger role, similar sets of correlation analyses could be produced, with a possibility of different results. In general, a correlation may not exist in Ottawa, but may exist in other cities, and the availability of such simulation platform could help to differentiate which cities have a public transit system contributing to pandemic spread. Furthermore, generalizing the study to more population-dense areas can also help to address sustainability objectives affected by public transportation decisions amid COVID-19 [28].

\section{Augmenting with real time sensory data}

With the advent of the internet of things (IoT) and sensor data and mobile edge computing technologies [29], it is possible to equip public transportation vehicles with anonymous sensors to detect when passengers board or exit a bus. This could be combined with other data when available, such as transit card usage and mobile crowd-sensing data to improve the model accuracy for better assessment capabilities. Furthermore, using the simulated results as training and the real sensor data as testing, the proposed simulation platform to track social contacts could be transformed into a reinforcement machine learningbased model that can predict ahead of time the contact risk of a particular public transit system, and whether it would be correlated to pandemic spread as there is significant room to leverage artificial intelligence and integrate it with exit strategies from the pandemic [30].

\section{Improvements via external factors}

The simulation capability produces credible results but indicates that the topography of public transportation systems can be insufficient for predicting community infections under some circumstances. The simulation could be augmented to take into account more inputs or external factors in those situations. 


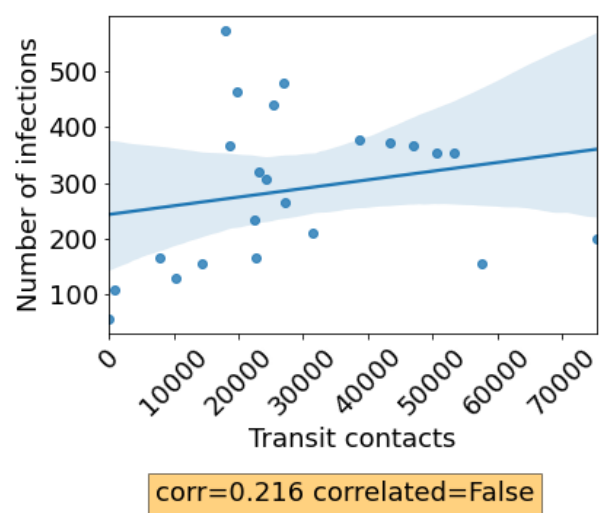

(a) $(\mu, \rho)=(5 \mathrm{~km}, 3 \mathrm{~km})$

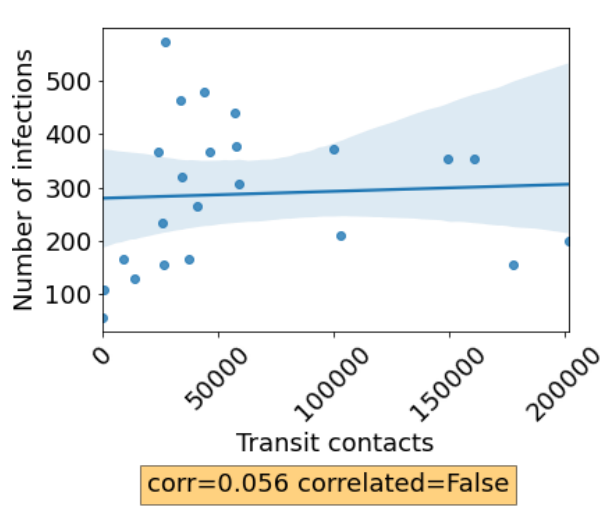

(b) $(\mu, \rho)=(10 \mathrm{~km}, 3 \mathrm{~km})$

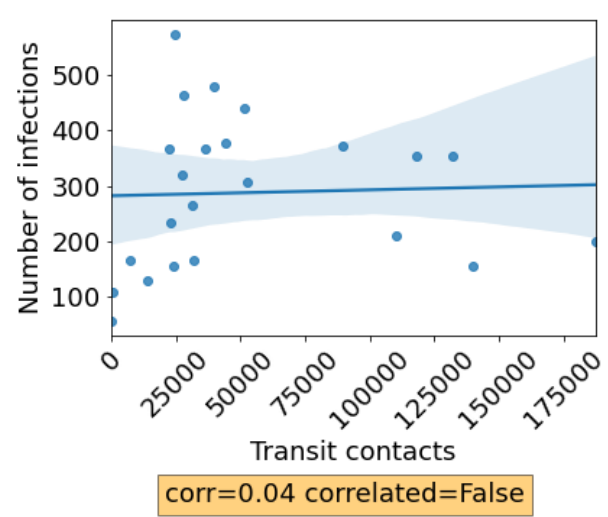

(c) $(\mu, \rho)=(10 \mathrm{~km}, 6 \mathrm{~km})$

Fig. 8: Comparison per ward between number of recorded COVID-19 infections and number of transit contacts recorded by the simulator under varying mean and standard deviation pairs $(\mu, \rho)$ for the trip length of an average passenger.

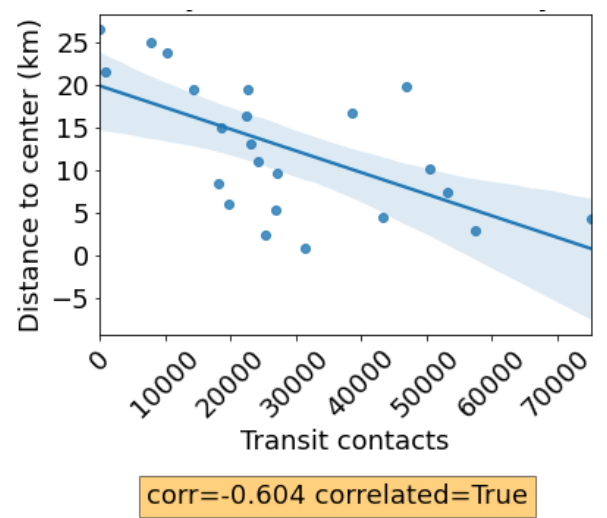

(a) $(\mu, \rho)=(5 \mathrm{~km}, 3 \mathrm{~km})$

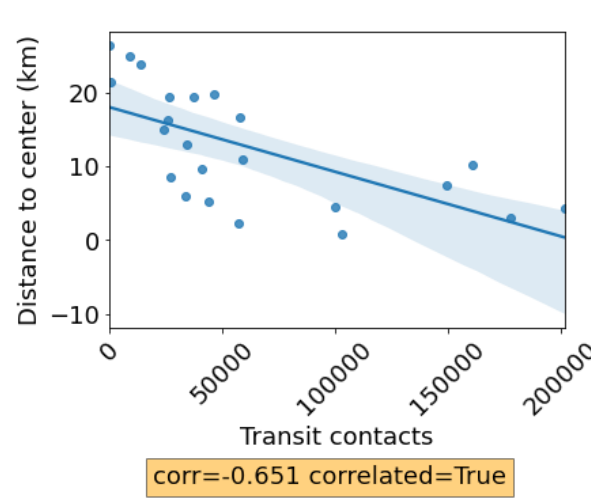

(b) $(\mu, \rho)=(10 \mathrm{~km}, 3 \mathrm{~km})$

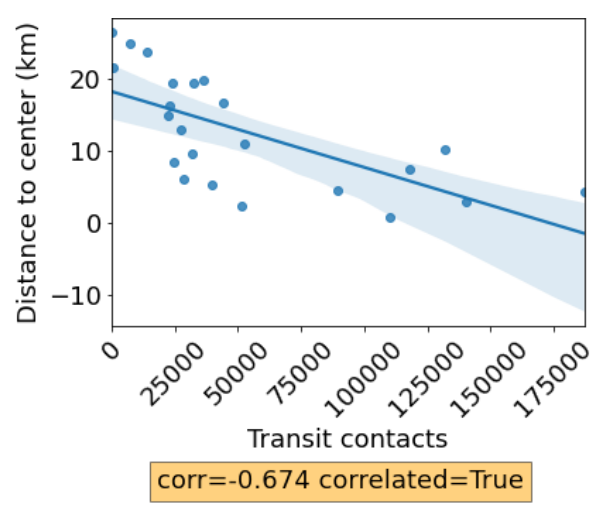

(c) $(\mu, \rho)=(10 \mathrm{~km}, 6 \mathrm{~km})$

Fig. 9: Comparison per ward between distance of ward to city center and number of transit contacts recorded by the simulator under varying mean and standard deviation pairs $(\mu, \rho)$ for the trip length of an average passenger.

One example is the physical proximity of passengers in public transportation vehicles. Currently, the simulator is designed with the assumption that being on the same public vehicle is a point of contact, whereas in reality the contact model is more chaotic, as it is still uncertain which surfaces are touched and how close passengers sit / stand during their commute, although preliminary studies on social distancing exist [31]. With an acquisition of data about vehicle layouts and passenger behaviour, as well as information on vehicle-route-schedule mapping information, invehicle actions can also be simulated to obtain a finer granular contact score. It is worth noting that external factors, such as local traffic, may also affect transit network performance as it would affect the duration of social contacts.

Inclusion of more data from/about a transit system enables intelligent models such as machine learning algorithms or neural network-based structures to augment the current rider behaviour's realism even further, to potentially produce more accurate results.

\section{CONCLUSION}

In this article, we have proposed a simulation framework which combines publicly available and existing data, including GTFS, Census and Apple mobility data, to simulate and predict the number of contacts that occur in a city due to public transit. We have ran simulations using real data from Ottawa, Ontario, Canada as a case study. We then produced simulated results at the ward level for a given time span, and analysed the outputs with known real-world infection numbers in the city for correlation.

For the city under study, the research results have been shown to be in-line with the current recommendations of public transportation research, showing that public transit ridership alone does not have a strong correlation to COVID19 transmission. Indeed, the simulation platform could easily be generalized to other cities, and would benefit from further testing.

This work is also open to extension, with augmentation from 
more data sources, or possibly combination with other research streams to provide even further insights into the role of public transportation in pandemics.

In a post-COVID-19 world, the approach of using public transportation data, combined with commuter behaviour will become a critical tool to provide citizens with a stronger understanding of pandemics and their relation to public transit while assisting the public transportation departments and municipalities in their planning of safer / healthier services to the public.

\section{ACKNOWLEDGEMENT}

This work was supported in part by the the Natural Sciences and Engineering Research Council of Canada (NSERC) under Grant Number ALLRP 552696-2020, and Mitacs Research Training Award. The authors would also like to express their appreciation to healthcare professionals and essential workers who have been at the front line of the battle against COVID-19.

\section{REFERENCES}

[1] X. Chen, "Infectious disease modeling and epidemic response measures analysis considering asymptomatic infection," IEEE Access, vol. 8, pp. 149 652-149660, 2020.

[2] S. Ishida, "Perspectives on supply chain management in a pandemic and the post-covid-19 era," IEEE Engineering Management Review, vol. 48, no. 3, pp. 146-152, 2020.

[3] K. Nikolopoulos, S. Punia, A. Schäfers, C. Tsinopoulos, and C. Vasilakis, "Forecasting and planning during a pandemic: Covid-19 growth rates, supply chain disruptions, and governmental decisions," European Journal of Operational Research, vol. 290, no. 1, pp. 99-115, 2021.

[4] A. Malik, S. Sinha, and S. Goel, "The "screen"ing of you and me: Effects of covid-19 on counterproductive work behaviors," IEEE Engineering Management Review, vol. 48, no. 3, pp. 37-43, 2020.

[5] S. Jamaludin, N. A. Azmir, A. F. Mohamad Ayob, and N. Zainal, "Covid19 exit strategy: Transitioning towards a new normal," Annals of Medicine and Surgery, vol. 59, pp. 165-170, 2020.

[6] M. Abdullah, C. Dias, D. Muley, and M. Shahin, "Exploring the impacts of covid-19 on travel behavior and mode preferences," Transportation Research Interdisciplinary Perspectives, vol. 8, p. 100255, 2020.

[7] "Cdc activities and initiatives supporting the covid-19 response and the president's plan for opening america up again," Centers for Disease Control and Prevention, May 2020. [Online]. Available: https: //www.cdc.gov/media/releases/2020/s0520-cdc-resources-open.html

[8] R. Vickerman, "Will covid-19 put the public back in public transport? a uk perspective," Transport Policy, vol. 103, pp. 95-102, 2021.

[9] S. Schwartz, "Public transit and covid-19 pandemic: Global research and best practices," https://www.apta.com/wp-content/uploads/APTA_Covid_ Best_Practices_09.29.2020.pdf, Sep 2020.

[10] L. Budd and S. Ison, "Responsible transport: A post-covid agenda for transport policy and practice," Transportation Research Interdisciplinary Perspectives, vol. 6, p. 100151, 2020.

[11] H. Dong, S. Ma, N. Jia, and J. Tian, "Understanding public transport satisfaction in post covid-19 pandemic," Transport Policy, vol. 101, pp 81-88, 2021.

[12] A. Tirachini and O. Cats, "Covid-19 and public transportation: Current assessment, prospects, and research needs," Journal of Public Transportation, vol. 22, 072020.

[13] E. Suryani, R. A. Hendrawan, P. F. Adipraja, A. Wibisono, B. Widodo, and R. Indraswari, "Modelling and simulation of transportation system effectiveness to reduce traffic congestion: a system dynamics framework," Transportation Planning and Technology, vol. 43, no. 7, pp. 670-697, 2020. [Online]. Available: https://doi.org/10.1080/03081060.2020.1805543

[14] A. V. Malang, L. R. S. Manlapas, P. M. B. Rivera, and M. N. Young, "Application of integer programming in maximizing of public utility bus passengers during covid-19 pandemic in select bus stops in epifanio delos santos avenue (edsa)," in 2020 7th International Conference on Frontiers of Industrial Engineering (ICFIE), 2020, pp. 135-141.
[15] Y. Shen, D. Guo, F. Long, L. A. Mateos, H. Ding, Z. Xiu, R. B. Hellman, A. King, S. Chen, C. Zhang, and H. Tan, "Robots under covid-19 pandemic: A comprehensive survey," IEEE Access, vol. 9, pp. 1590-1615, 2021.

[16] Y. Amir, S. Deb, M. S. Alam, Y. Rafat, and S. Hameed, "Real world solutions for smart cities transportation to be pandemic ready," in 2020 Fifth International Conference on Research in Computational Intelligence and Communication Networks (ICRCICN), 2020, pp. 159-164.

[17] P. Gaudette, R. Chapleau, and T. Spurr, "Bus network microsimulation with general transit feed specification and tap-in-only smart card data," Transportation Research Record, vol. 2544, no. 1, pp. 71-80, 2016. [Online]. Available: https://doi.org/10.3141/2544-09

[18] K. Farkas, G. Feher, A. Benczur, and C. Sidlo, "Crowdsending based public transport information service in smart cities," IEEE Communications Magazine, vol. 53, no. 8, pp. 158-165, 2015.

[19] S. Kielienyu, B. Kantarci, D. Turgut, and S. Khan, "Bridging predictive analytics and mobile crowdsensing for future risk maps of communities against covid-19," in Proceedings of the 18th ACM Symposium on Mobility Management and Wireless Access, ser. MobiWac '20. New York, NY, USA: Association for Computing Machinery, 2020, p. 37-45. [Online]. Available: https://doi.org/10.1145/3416012.3424624

[20] A. Boukerche, B. Kantarci, and C. Kaptan, "Towards ensuring the reliability and dependability of vehicular crowd-sensing data in gps-less location tracking," Pervasive and Mobile Computing, vol. 68, p. 101248, 2020.

[21] (2019, Jun) Census profile, 2016 census canada. [Online]. Available: https://www12.statcan.gc.ca/census-recensement/2016/dp-pd/ prof/details/page.cfm?Lang=E

[22] S. Temiz and D. G. Broo, "Open innovation initiatives to tackle covid-19 crises: Imposter open innovation and openness in data," IEEE Engineering Management Review, vol. 48, no. 4, pp. 46-54, 2020.

[23] A. Nikiforova, "Timeliness of open data in open government data portals through pandemic-related data: a long data way from the publisher to the user," in 2020 Fourth International Conference on Multimedia Computing, Networking and Applications (MCNA), 2020, pp. 131-138.

[24] (2019, Aug) Ward boundaries 2010. [Online]. Available: https: //open.ottawa.ca/datasets/wards-2010

[25] L. Liu, H. Miller, and J. Scheff, "The impacts of covid-19 pandemic on public transit demand in the united states," PLoS ONE, vol. 15, 112020.

[26] Covid-19 maps for ottawa. [Online]. Available: https://open.ottawa.ca/ datasets/data-tables-for-public-covid-19-maps

[27] Rns express research needs statements specific to transportation and pandemics. [Online]. Available: http://www.trb.org/main/ CallforRNSTransportationAndPandemics.aspx

[28] X. Tian, C. An, Z. Chen, and Z. Tian, "Assessing the impact of covid-19 pandemic on urban transportation and air quality in canada," Science of The Total Environment, vol. 765, p. 144270, 2021.

[29] A. Ksentini and B. Brik, "An edge-based social distancing detection service to mitigate covid-19 propagation," IEEE Internet of Things Magazine, vol. 3, no. 3, pp. 35-39, 2020.

[30] M.-H. Tayarani N., "Applications of artificial intelligence in battling against covid-19: A literature review," Chaos, Solitons Fractals, vol. 142, p. 110338, 2021.

[31] E. K. J. Dzisi and O. A. Dei, "Adherence to social distancing and wearing of masks within public transportation during the covid 19 pandemic," Transportation Research Interdisciplinary Perspectives, vol. 7, p. 100191, 2020. 